\title{
Histoire des transports, histoire culturelle : mobiliser le passé des chemins de fer
}

Transport history, cultural history: mobilizing the railways' past

\section{Colin Divall}

\section{OpenEdition}

\section{Journals}

Édition électronique

URL : https://journals.openedition.org/rhcf/1040

DOI : 10.4000/rhcf.1040

\section{Éditeur}

Rails \& histoire

Édition imprimée

Date de publication : 1 juin 2008

Pagination : 313-326

ISSN : 0996-9403

Référence électronique

Colin Divall, « Histoire des transports, histoire culturelle : mobiliser le passé des chemins de fer », Revue d'histoire des chemins de fer [En ligne], 39 | 2008, mis en ligne le 01 juin 2011, consulté le 22 avril 2022. URL : http://journals.openedition.org/rhcf/1040 ; DOI : https://doi.org/10.4000/rhcf.1040 
Colin DIVALL

\title{
Histoire des transports, histoire culturelle : mobiliser le passé des chemins de fer
}

Traduit de I'anglais par Marie-Noëlle Polino

\begin{abstract}
$\int^{2}$
e commence par féliciter l'Association pour l'histoire des chemins de fer en France d'avoir atteint son vingtième anniversaire, et par remercier le professeur Caron, Marie-Noëlle Polino et leurs collègues pour leur aimable invitation qui me donne l'occasion de m'adresser à vous cet après-midi. L'AHICF est une des plus importantes institutions européennes dédiées à la recherche en histoire des chemins de fer et ce qu'elle a réussi à accomplir pendant ces deux dernières décennies a fait une profonde impression sur les chercheurs hors de France. Les historiens, dont certains sont fort éminents, qui ont fait de l'histoire des chemins de fer en France le domaine de recherche vivant et stimulant qu'il est aujourd'hui sont nombreux. Cependant, sans le travail d'organisation effectué par l'AHICF en coulisses - qu'il s'agisse de colloques comme celui-ci, de la publication de la Revue d'histoire des chemins de fer et d'autres ouvrages, de l'aide apportée aux chercheurs et aux étudiants pour se rencontrer, de la constitution de fonds documentaires, de la recherche de financements - les efforts individuels de ces chercheurs auraient eu bien moins de résultats et peut-être n'auraient-ils pas été aussi connus qu'ils le sont dans d'autres parties du monde.
\end{abstract}


L'AHICF a également apporté un soutien généreux à des initiatives qui dépassaient l'espace français, la plus importante étant peut-être le programme de recherche de cinq ans (2000-2005) d'envergure européenne «COST» (coopération scientifique et technique) «Vers un réseau de transport européen intermodal : les leçons de l'histoire ». L'AHICF est le type d'organisation dont on n'imagine pas pouvoir se passer, même si dans la plupart des pays nous devons nous débrouiller comme nous le pouvons. Dans tous les cas j'attends avec impatience et plaisir de pouvoir apprécier les résultats des vingt prochaines années de travail de l'AHICF.

\section{L'histoire des transports est-elle en train de faire son " tournant culturel "?}

Je souhaite aujourd'hui mettre l'accent sur quelques tendances récentes en historiographie des transports, dans l'espoir de suggérer par elles les possibles axes de développement de la recherche en histoire des chemins de fer.

Je m'appuie en partie sur un article de réflexion que j'ai écrit à la mi2004 avec mon collègue George Revill, un représentant de la géographie historique anglaise, et qui a été publié par le Journal of Transport History en mars $2005^{1}$. Cet article a donné lieu à discussion et vous pouvez suivre le débat, qui continue à rebondir, dans les colonnes du JTH. Par ailleurs, une version française revue et corrigée de ce travail devrait être publiée dans un recueil sous presse intitulé : De l'histoire des transports à l'bistoire de la mobilité2.

Ce que George et moi suggérons principalement, c'est que les historiens des transports devraient être beaucoup plus attentifs aux interactions complexes entre la «culture » et la façon dont la mobilité des gens, des choses et des idées a été historiquement mise en œuvre par les sociétés. Cette idée fondamentale, à savoir prendre en compte les aspects culturels du transport, ne prétend certes pas à l'originalité. Un petit nombre d'historiens, et pas seulement dans le monde anglophone, la mettent en pratique depuis déjà longtemps.

1- "Cultures of transport. Representation, practice and technology ", The Journal of Transport History, 26/1, mars 2005, p. 99-111. Voir par la suite : Michael Freeman, " "Turn if you want to": a comment on the "cultural turn" in Divall and Revill's "Cultures of transport" ", The Journal of Transport History, 27/1 (mars 2006), p. 138-143 ; C. Divall et G. Revill, « No turn needed: a reply to Michael Freeman », The Journal of Transport History, $27 / 1$ (mars 2006), p. 144-149.

2- Colin Divall et George Revill, «Les cultures du transport : représentation, pratique et technologie ", in Vincent Guigueno et Mathieu Flonneau (dir.), De l'bistoire des transports à l'bistoire de la mobilité, Rennes, Presses universitaires de Rennes, 2009. 
L'Histoire des voyages en train de Wolfgang Schivelbusch, publié d'abord en allemand en 1977 et traduit en anglais deux ans plus tard (The Railway Journey: The Industrialization of Time and Space in the 19th Century) [en français en 1990 seulement] $]^{3}$, n'a certainement pas été le premier ouvrage à se demander comment l'avènement des chemins de fer avait été perçu et représenté par les arts, académiques comme populaires. S'il a eu de l'importance, c'est parce qu'il alliait une analyse très fine de l'expérience subjective nouvelle qu'était le voyage en train avec une sensibilité à ses fondements matériels qui résident dans la technique. Bien que le cadre conceptuel qui permet à Schivelbusch de lier ces deux champs pose problème, son livre anticipe de bien des façons ce que George et moi souhaitions dire dans notre article. Beaucoup plus récemment, d'autres auteurs comme le regretté Jack Simmons (en 1991) ${ }^{4}$, Michael Freeman $(\text { en 1999) })^{5}$ et Ian Carter (en 2001) $)^{6}$, pour se limiter à ces trois noms, ont aussi analysé divers aspects de la culture des chemins de fer, sans toujours adopter une perspective théorique aussi radicale que celle de Schivelbusch.

\section{Alors, c'est quoi la " culture" ?}

Cet ensemble de travaux a évolué très rapidement ces dernières années, au point qu'aucun historien sérieux ne peut aujourd'hui comprendre la «culture » comme la seule représentation esthétique du transport et du voyage dans les arts académiques et populaires - peinture, musique, cinéma, affiches, dessins humoristiques, danse, romans, poésie, etc. Ce terme englobe beaucoup d'autres choses. Il fait référence aux valeurs, normes, croyances et attitudes qui donnent du sens, en particulier un sens symbolique (c'est-à-dire non fonctionnel), à d'autres personnes, à des entités et processus sociaux, et aux objets matériels et au cadre de notre vie quotidienne. Aujourd'hui, par exemple, nous avons encore tendance à penser la rue - quelle qu'en soit la réalité ! - comme un espace de circulation dans lequel les flux de véhicules motorisés doivent avoir la première place et s'écouler librement. Ces significations peuvent être exprimées par les arts, mais elles sont aussi sous-jacentes dans la façon dont nous vivons notre vie quotidienne. Et parce que ces significations sont partagées à un certain degré par les membres d'une société donnée, elles fonctionnent comme « une ressource pour la pratique » pour l'organisation de la vie quotidienne,

3- Wolfgang Schivelbush, Histoire des voyages en train, Paris, Le Promeneur, 1990, traduit de l'allemand par Jean-François Boutout, 252 pages.

4- The Victorian Railway, Londres, Thames and Hudson, 1991, 416 pages.

5- Railways and the Victorian Imagination, New Haven et Londres, Yale University Press, 1999, 264 pages.

6- Railways and Culture in Britain: the epitome of modernity, Manchester, Manchester University Press, 2001, 352 pages. 
mais aussi comme un moyen de fonder le sentiment d'une identité collective. Il n'y a bien sûr rien de neuf dans cette définition élargie du concept de « culture ». Elle est depuis longtemps un lieu commun du discours universitaire et est désormais si familière qu'on la retrouve dans les éditions récentes des dictionnaires de langue anglaise : j'imagine qu'il en est de même depuis longtemps en français.

C'est cette culture-là qui devrait jouer un rôle plus large dans la recherche en histoire des chemins de fer et dans l'écriture de cette histoire. Car tout transport est un acte profondément culturel. Le processus physique de se déplacer d'un point $\mathrm{A}$ à un point $\mathrm{B}$ (sa dimension matérielle, fonctionnelle, instrumentale) est imprégné de significations inscrites dans la société (sa dimension symbolique et l'expression de celle-ci), comme par exemple « je suis libre quand je suis au volant » [driving is freedom] : ce sont là des cultures du transport. La meilleure façon de penser ces cultures du transport est de les considérer comme des processus sociaux, des processus par lesquels des significations ont été rattachées à des formes particulières de mobilité, et par lesquels des modèles particuliers de mobilité - ou de non-mobilité - se sont historiquement formés, stabilisés et, dans bien des cas, ont évolué pour finalement se décomposer.

Le terme « automobilisme » rend compte, du moins en anglais, de cette combinaison d'une fonction pratique [motoring veut aussi dire " conduite »] avec un ensemble tout aussi important de valeurs symboliques plus larges, qui incluent bien entendu le statut social acquis par la possession d'un bien de consommation onéreux. C'est en accordant davantage d'attention à ces processus de formation des cultures et d'inscription de ces cultures dans la société que nous autres historiens des chemins de fer pourrions arriver à mieux comprendre les modes et les causes de l'évolution de la mobilité ferroviaire.

\section{Est-ce ce vraiment une nouveauté ?}

Étant donné que cette idée fondamentale de la «culture » nous est si familière, il n'est pas surprenant que quelques critiques disent qu'il n'y a rien de réellement nouveau dans ce que G. Revill et moi-même avons avancé. Je suis heureux de reconnaître le bien-fondé de ce reproche, quand je vois le nombre d'articles publiés par le leader des revues d'histoire des transports en langue anglaise, le Journal of Transport History, qui incluent désormais cette notion élargie de la culture. Mais je ne suis pas convaincu que tous les historiens des transports, y compris ceux qui font profession d'écrire sur les cultures du transport, soient aussi clairs et déterminés dans leurs analyses que je pourrais le souhaiter. 


\section{Quelques avancées récentes...}

Permettez-moi de tenter d'expliquer ce que j'entends par un rapide examen de ce qui est dit et de ce qui ne l'est pas dans la bibliographie la plus récente en langue anglaise.

Ce qui va suivre n'a rien bien entendu d'une enquête exhaustive. Mais il est clair qu'il y a eu un déplacement de l'intérêt des chercheurs vers l'analyse de la représentation du transport et de la mobilité par divers media. J'utilise ici des représentations visuelles parce qu'il m'est plus facile de le faire, non parce que je considère les images comme le seul sujet qui doive nous intéresser. Cependant, comme vous pouvez le voir, et c'est un élément du fait qu'ils se tournent vers la culture comme ressource quotidienne, les historiens se sont dirigés davantage vers des représentations qui relèvent plutôt, $d u$ moins relativement, du banal que des beaux-arts. Il peut s'agir de résultats d'une commande des sociétés de transport, comme cet exemple d'une publicité de l'entre-deux-guerres ; elles peuvent être encore plus vernaculaires et exprimer un ensemble de significations propre à un individu ou à un groupe social particulier. Ce dessin, tiré de l'un des magazines des compagnies de chemin de fer pendant l'entre-deux-guerres, est, pour autant que je puisse le dire, l'œuvre d'un cheminot (fig. 1).

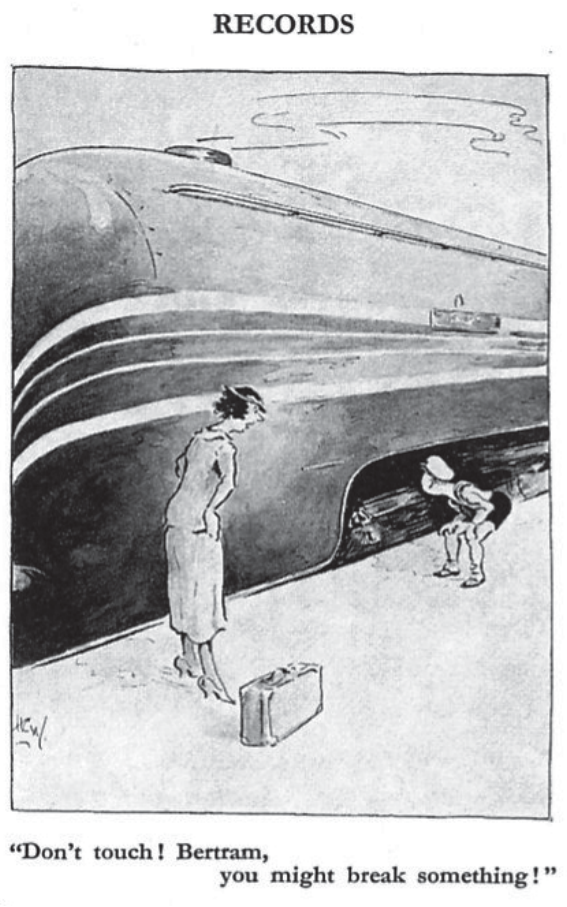

- Figure 1. "Ne touche à rien, Bertrand, tu vas casser quelque chose. "

La déconstruction des significations de ces sortes d'images et d'expressions analogues dans d'autres media fait à présent partie intégrante du travail de l'historien des transports. Comme je l'ai déjà laissé entendre, établir des connexions entre les significations inscrites dans les images, leurs auteurs et les institutions plus larges qui les ont produites et, quand c'est possible, ce qui n'est pas fréquent, avec les publics qui les ont interprétées devrait être mis en lien soit avec l'idée, soit avec la pratique de la mobilité quotidienne. J'insiste sur le fait que le point important ici est le fait que la façon dont les gens se sont 
déplacés dans le passé et les raisons qu'ils avaient de le faire ont été informées en partie - mais seulement en partie - par la façon dont ces mêmes gens attribuaient une signification symbolique à diverses formes de transport.

Les travaux les plus intéressants dans ce courant prennent leur origine dans la reconnaissance du fait que la mobilité personnelle, en particulier au siècle dernier [le $\left.\mathrm{XX}^{\mathrm{c}}\right]$, est devenue une sorte de consommation; le fait de se déplacer n'était pas seulement une affaire de nécessité mais aussi, du moins parfois, quelque chose que l'on pouvait faire par choix, et qui pouvait contribuer à exprimer un statut social ou un sentiment d'identité individuelle.

Beaucoup a déjà été fait pour analyser en ces termes l'automobilisme, pas seulement aux États-Unis mais aussi et de plus en plus au Royaume-Uni, en Allemagne et ailleurs en Europe. L'idée nous est par exemple devenue familière d'une phase des pionniers de l'automobilisme avant la Première Guerre mondiale souvent associée au genre masculin, lié aux risques attachés à la conduite d'une machine puissante. Mais dans le contexte britannique, la notion de consommation est de plus en plus souvent appliquée aux chemins de fer, en particulier, mais pas seulement, quand on en vient à déconstruire les représentations associées aux voyages de loisir. Nous pouvons envisager utilement de parler de « railing » pour caractériser la mobilité par rail, comme nous appelons l'automobilisme «motoring». Mais que cette terminologie soit ou non adoptée, les chercheurs mettent déjà en œuvre les idées qu'elle tente d'exprimer. Par exemple, une image comme celle de ce couple d'âge mûr voyageant dans une voiture du réseau London, Midland and Scottish Railway des années 1930 nous en dit beaucoup sur la façon dont les chemins de fer tentaient de concurrencer l'automobile en vendant une image de confort presque domestique et de respectabilité (fig. 2). Je reviendrai brièvement aux voitures de chemin de fer tout à l'heure.

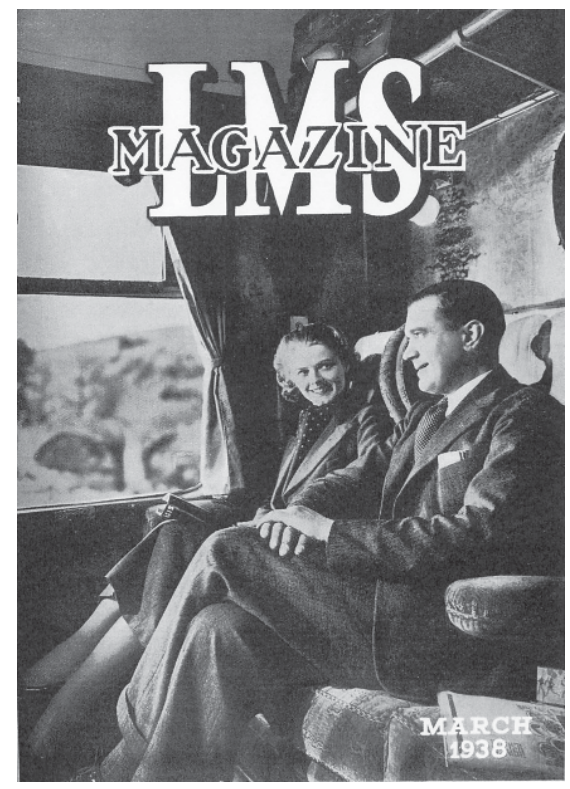

• Figure 2 


\section{Qu'est-ce qui fait le lien entre représentation et pratique?}

La représentation du transport est une chose, sa consommation quotidienne comme mobilité en est une autre. Qu'est-ce qui fait le lien entre les deux ? La signification symbolique est certainement une partie de la réponse, mais les significations symboliques ne flottent pas librement dans un éther indéterminé. Elles sont toujours enracinées dans des réalités matérielles. Voilà pourquoi la technique doit être un aspect central - et peut-être même le plus important - de la réalité matérielle que doivent prendre en considération les historiens qui analysent les cultures du transport. Je comprends ici la «technique » dans des termes très larges, pour y inclure non seulement le «hardware » - véhicules et infrastructures - mais aussi les institutions sociales qui leur permettent de fonctionner ensemble, c'est-à-dire le système socio-technique. C'est la combinaison des trois qui fait un système de transport. De plus ces éléments ont pour liant les significations que nous leur donnons, les valeurs culturelles qu'à la fois nous mettons en œuvre et reproduisons, et que nous transformons parfois chaque fois que nous " performons » l'acte de nous déplacer. Cette perspective théorique fait entrer l'histoire des transports dans une sémiotique sociale plus large, qui déconstruit les objets du quotidien, les environnements construits et les actions de tous les jours qui s'y passent en même temps que les représentations plus intentionnelles que nous avons déjà examinées.

\section{La technique est-elle un facteur nouveau?}

Est-ce nouveau ? C'est peut-être sur ce point que G. Revill et moimême nous nous distinguons d'un nombre significatif, quoique en rapide diminution, de chercheurs qui se considèrent d'abord comme des historiens des transports ou de la mobilité. Certains d'entre eux ne prennent pas la technique suffisamment au sérieux. Ce n'est pas nécessairement parce qu'ils l'ignorent, c'est parce qu'ils s'en font une idée beaucoup trop étroite. John Walton, l'historien social du tourisme, montre cela très bien. Ses remarques font apparaître combien l'histoire des techniques a été pendant longtemps au mieux réduite à un processus qui peut être expliqué en termes économiques? ${ }^{7}$. L'économie est bien sûr un aspect extrêmement important du changement technique. Mais réciproquement, et j'espère l'avoir à présent clairement montré, il ne suffit pas de penser les techniques de transport comme seulement des phénomènes d'ordre économique. Elles sont investies de significations, et font intégralement partie de la culture si on la comprend comme « le socle partagé par tous de

7- John Walton, « Transport, travel, tourism and mobility: a cultural turn? ", The Journal of Transport History, 27/2 (sept. 2006), p. 129-134. 
l'action sociale ». Pour faire bref, elles sont des éléments de la culture matérielle. Ce serait bien mal de ma part de laisser entendre qu'aucun historien ne l'a reconnu avant moi ! J'ai déjà mentionné l'œuvre de Wolfgang Schivelbusch, et il n'est pas difficile de penser à d'autres chercheurs de renom, comme l'Américain David Nye, qui ont analysé la façon dont les techniques de transport ont évolué historiquement comme des cultures matérielles. Les sociologues des techniques - Bruno Latour étant sans doute le plus éminent ici à Paris - ont aussi suivi ces orientations. Cependant aucun n'est un historien des chemins de fer à l'origine, et jusqu'à récemment leur influence ne s'est pas fait très fortement sentir sur nous. Cependant, quand j'écrivais voici sept ans : « les historiens des transports n'approfondissent que bien rarement les voies par lesquelles les objets techniques en viennent à incarner des significations sociales, et [ne pensent guère] la façon dont a évolué l'interaction entre ces objets, les individus et les structures sociales ${ }^{9}$, les chercheurs qui travaillent dans le domaine de l'histoire des techniques, en particulier l'école qui est derrière la revue Technology and Culture, s'intéressaient de plus en plus à la façon dont les significations symboliques et la matérialité des techniques de transport se donnaient les unes aux autres leur forme et leur définition. Ma remarque, si elle n'est pas aussi pertinente aujourd'hui qu'elle ne le fut, n'a pas cependant perdu toute sa force. Nous autres historiens des chemins de fer ferions bien de regarder du côté de l'histoire des transports urbains, un champ en pleine ébullition qui produit des travaux à la fois théoriquement valables et nuancés du point de vue de l'expérience qui montrent comment les valeurs et les attitudes des gens envers la mobilité quotidienne ont été informés par - et à leur tour ont déterminé - les véhicules et autres techniques de transport qu'ils utilisaient. Ce genre de recherche a un arbre généalogique fourni, et l'étude comparative faite par John P. McKay voici maintenant trente ans entre les conceptions esthétiques européennes et nordaméricaines et leur impact sur l'électrification des tramways et, de là, sur la mobilité urbaine vaut encore la lecture ${ }^{10}$. De la même façon, l’ouvrage publié en 1994 par Clay McShane sur l'émergence de l'automobilisme dans les villes américaines montre comment des évolutions dans la technologie du véhicule à moteur et celle des routes allèrent de pair avec celles des significations qui leur étaient

8- Voir ses ouvrages principaux : Electrifying America. Social Meanings of a New Technology, 18801940, Cambridge, MIT Press, 1990 et American Technological Sublime, ibid., 1994.

9- « Historians of transport rarely think very deeply about the ways in which technological things came to embody social meaning, and how the interplay between these objects, individuals and social structures changed ", C. Divall et Andrew Scott, Making Histories in Transport Museums, Londres et New York, Leicester University Press, 2001, 221 pages, p. 119.

10- Tramways and Trolleys: The Rise of Urban Mass Transport in Europe, Princeton University Press, 1976. 
attribuées ${ }^{11}$. On se mit à penser les rues comme un axe de pénétration dans un espace plus large de circulation urbaine plutôt que comme un espace public ouvert et un lieu pour la vie du quartier ; les véhicules à moteur furent considérés comme un moyen de libération et un symbole du statut social : «la technique, ainsi améliorée, était pour une large part une construction sociale.»

\section{Mobiliser le passé des chemins de fer}

De quelle façon les historiens des chemins de fer pourront-ils continuer à mettre en œuvre ces types d'approches dans les années à venir ? Pour récapituler, j’ai déjà proposé de penser le transport par chemin de fer comme une forme de mobilité de même nature que l'automobilisme ( railing »). Cette mobilité ferroviaire a dû être historiquement produite - c'est l'aspect le mieux étudié par les recherches actuelles - mais aussi consommée par les utilisateurs. Nous avons besoin de davantage de recherches sur la consommation du voyage en train, mais avant tout nous avons besoin de faire le lien entre production et consommation. La technique est au cœur de tout cela : la mobilité ferroviaire est un processus technologique, ou, plus complètement, un processus technico-culturel, qui est créé, lancé sur le marché et vendu par les compagnies et leur personnel, et qui est acheté et utilisé par des clients qui sont aussi des consommateurs.

Permettez-moi de revenir rapidement à la technologie de la voiture de chemin de fer. Des historiennes comme Barbara Schmucki, Amy Richter et Barbara Ann Welke insistent sur le fait que des véhicules de transport public comme les remorques de tramways et les voitures de chemins de fer étaient des espaces dans lesquels les notions genrées des comportements acceptables ou non pour l'un et l'autre sexe étaient mises en cause ${ }^{12}$; ces notions informaient l'usage que les femmes (et les hommes) faisaient de ces techniques de mobilité, usage qui en retour les modifiaient. Voyons en quelques mots comment le compartiment de chemin de fer a été investi du point de vue du genre en Grande-Bretagne pendant l'entre-deux-guerres. Sheryl Kroen a pour thèse que l'amélioration de « la reconnaissance du consommateur comme un agent actif de la démocratisation » au début du $\mathrm{XX}^{\mathrm{e}}$ siècle était en partie l'effet de l'affirmation des droits des femmes à un statut politique, économique et social

11- Down the Asphalt Path: American Cities and the Automobile, New York, Columbia University Press, 1994.

12- Barbara Schmucki, «On the trams: women, men and urban public transport in Germany », The Journal of Transport History, 23/1 (mars 2002), p. 60-72 ; Amy Richter, Home on the Rails: Women, the Railroad, and the Rise of Public Domesticity, Chapel Hill, University of North Carolina Press, Gender \& American Culture Series, 2005 ; Barbara Ann Welke, Recasting American Liberty: Gender, Race, Law and the Railroad Revolution 1865-1920, Cambridge, Cambridge University Press, 2001, 405 pages. 
à part entière ${ }^{13}$. Cette analyse rejoint ce que nous savons du marketing des compagnies de chemin de fer britanniques pendant l'entre-deux-guerres. Il a été de plus en plus pensé pour attirer les femmes, comme le suggère cette photo d'un compartiment de chemin de fer vu comme un intérieur coquet, un espace de détente où l'on tient une conversation distinguée (fig. 3). D’un autre côté, nous pouvons aussi trouver des expressions des doutes masculins devant la place grandissante que prennent les femmes en tant que consommatrices dans l'espace public. Prenons par exemple ce dessin publié dans un magazine de l'une des compagnies de chemin de fer ("Perplexe!", août 1928), qui exprime le sentiment d'un malaise masculin devant l'effet dérangeant du changement intervenu dans les modes et le comportement féminins sur les conventions et le sens des convenances sociales en vigueur dans les chemins de fer (fig. 4).

Dans les années 1930 le même magazine publia également plusieurs dessins qui décrivaient les inconvénients du voyage en chemin de fer pour les utilisateurs, la violation, du point du genre, des normes du comportement bien élevé. Je relève deux catégories, d'abord une série de dessins du milieu des années 1930 qui dépeint le compartiment comme un espace permettant l'expression d'une certaine licence (hétéro)sexuelle. C'est en soi un thème certes éculé, mais le degré de contrôle de la situation affirmé par cette femme très sûre d'elle-même est moins habituel (fig. 5). Le deuxième dessin (octobre 1937) utilise l'idée d'un intérieur domestique pour connoter les qualités qui font désirer le chemin de fer, vitesse, confort, sécurité (fig. 6). Mais l'homme est allé trop loin dans son interprétation - et il est frappant de constater qu'au contraire de la première image où deux femmes voyageaient ensemble, dans ces deux derniers exemples nous sommes en $3^{e}$ classe. Comme toujours, la classe sociale et le genre interagissent.

Pour résumer, le compartiment de chemin de fer était déterminé du point de vue du genre par les responsables de la vente des chemins de fer et les cheminots d'un grand nombre de façons, c'est une évidence, pour inclure à la fois la « femme qui voyage » comme un consommateur du moins potentiel et les avantages (ou l'inverse) de la mobilité ferroviaire auprès d'un marché plus large. Que ces constructions n'aient pas toujours été en accord avec celles du marketing des compagnies suggère une recherche fructueuse qui explorerait la façon dont elles ont tenté de contrôler la déconnexion entre les messages qu'elles tentaient de faire passer et les savoirs plus complexes qui prévalaient chez les cheminots mais aussi plus largement dans la société.

13- A «positive appraisal of the consumer as an active agent of democratization », dans Sheryl Kroen, "A political history of the consumer», The Historical Journal, 47/3 (2004), p. 709-736, p. 720. 
APRIL

I935

Figure 3 •

Figure 4
Principal Contents:

CELL MANUFACTURE

MODERNISATION OF RUNNING SHEDS GRANGEMOUTH HARBOUR AND DOCKS LONDON

A HOME-MADE BIRD BATH

THOUGHTS AND COMMENTS

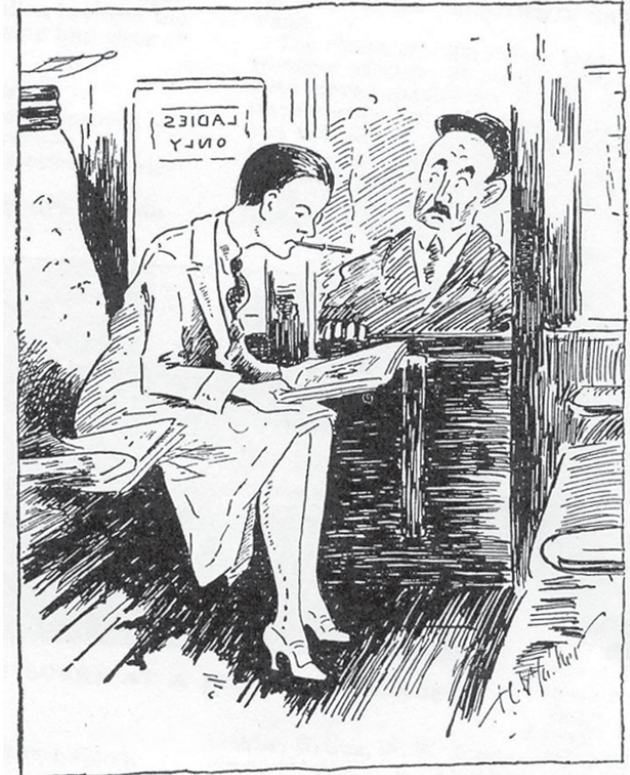

NONPLUSSED ! 


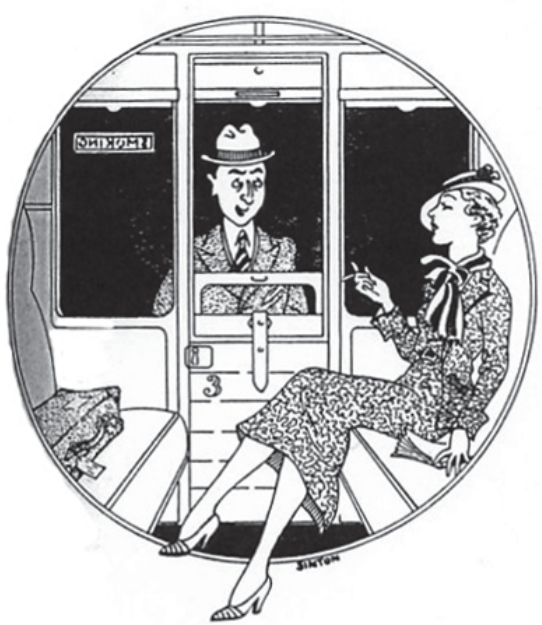

He (making final effort to form acquaintance) : "Pardon me! Did I leave anything behind ?" She : "You did! A decidedly poor impression."
Figure 5

Lui (faisant un dernier effort pour nouer connaissance): "Excusez-moi, ai-je laissé quelque chose? "

Elle : « Oui, une impression tout à fait déplorable. »

Figure 6 •

«Mon mari a l'habitude de sa cheminée, voyez-vous. »

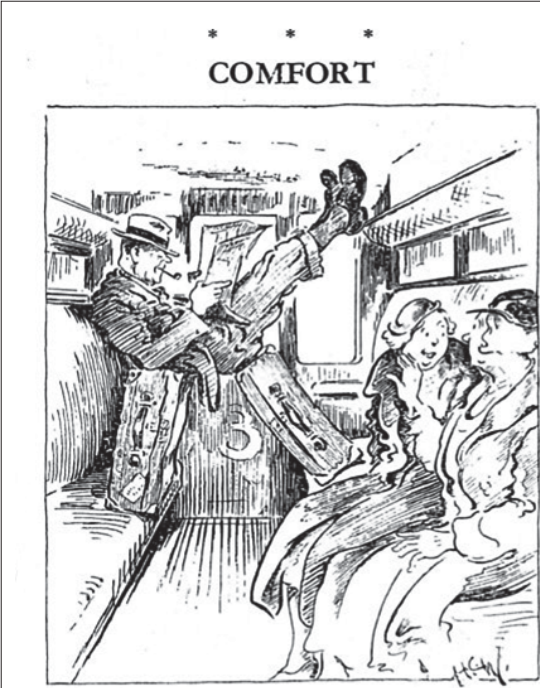

"You see, my husband's so partial to a mantel-shelf." 


\section{Finalement...}

J'ai parlé de quelques-uns des moyens par lesquels l'historiographie des chemins de fer pourrait évoluer en tirant parti d'une notion de la culture comprise comme un système de significations mises en œuvre quotidiennement. Le principal intérêt de cette approche, d'un point de vue intellectuel, est de faire progresser notre compréhension de la façon et des raisons pour lesquelles la mobilité ferroviaire, «railing» - l'idée et la pratique de la mobilité associées au déplacement en train -, a pris une telle importance dans des sociétés industrialisées comme la France et la Grande-Bretagne dans le passé, et de la façon et des raisons pour lesquelles cette mobilité est peu à peu tombée en disgrâce dans ces pays pendant le dernier siècle. Mais bien que nous passions la majeure partie de notre temps à écrire l'histoire des chemins de fer pour ellemême, cela vaut la peine de réfléchir quelques instants à la façon dont elle pourrait aussi servir d'autres objectifs sociétaux.

Le transport devient aujourd'hui une priorité dans les préoccupations politiques, ce qui laisse penser que si nous trouvons la façon appropriée de nous faire entendre, les historiens pourront trouver des publics prêts à les écouter en dehors du monde universitaire. Nous sommes nombreux à reconnaitre que l'augmentation de la mobilité est contraire au développement durable, et que les coûts et les avantages de la mobilité contemporaine ne sont pas distribués de manière équitable entre les groupes sociaux. Mais la mobilité des personnes n'est pas le seul souci. On doit aussi se préoccuper du transport de marchandises, d'autant davantage que la mondialisation augmente les distances parcourues par les matières premières et les produits manufacturés et que l'évolution des procédures tant de la consommation que de la production exige des livraisons « juste-à-temps » plus fréquentes qui concernent des espaces géographiques plus grands.

L'histoire peut-elle contribuer à guider l'élu, le responsable des politiques publiques ou même le citoyen ou la citoyenne qui a le souci d'influer le cours des choses en maintenant ou modifiant son propre comportement, un monde dans lequel les états-nations attachés à leur territoire sont, on peut le dire, moins importants que les réseaux et les flux mondiaux ? Je crois qu'elle le peut. L'histoire, bien sûr, n'est pas un guide gravé dans le marbre pour l'avenir. Mais si comprendre le passé ne peut pas déterminer l'avenir, cela peut cependant nous aider à appréhender, de façon large, les facteurs qui expliquent également la persistance ou le caractère éphémère de certains modèles de mobilité et, par là, en suggérant des modes appropriés d'intervention, à influencer la façon dont ces modèles évoluent. 
Une historiographie qui comprend les chemins de fer comme des cultures matérielles sera mieux placée pour le faire qu'une histoire qui ne s'autorise que des approches plus traditionnelles, quelle que soit l'importance que conservent celles-ci. 